\title{
Performance Evaluation of High-Resolution Ultrasound versus Magnetic Resonance Imaging in Diagnosing Peripheral Nerve Pathologies
}

\author{
${ }^{1}$ Department of Radiology, Fortis Escorts Hospital, Faridabad, \\ Haryana, India \\ ${ }^{2}$ Consultation Diagnostic and Intervention Radiology, JK Medicity \\ Super Speciality Hospital, Jammu, India \\ ${ }^{3}$ Department of Radiology, MRI Centre of GMSH, Chandigarh, \\ Punjab, India \\ ${ }^{4}$ Department of Radiology, Medanta-The Medicity, Gurugram, \\ Haryana, India
}

Neha Nischal ${ }^{1}$ Saurabh Gupta ${ }^{2} \quad$ Kanhaiya Lal $^{3} \quad$ Jatinder Pal Singh ${ }^{1}$

Address for correspondence Jatinder Pal Singh, DNB, DMRD, FRCR, Department of Radiology, Medanta-The Medicity, Sector 38, Gurugram, Haryana 122001, India (e-mail: jpsingh@doctors.org.uk).

Indian J Radiol Imaging 2021;31:43-48.

\begin{abstract}
Keywords

- high-resolution

- magnetic resonance

- neurography

- submillimeter

- ultrasound

Background High-resolution ultrasound (HRUS) and magnetic resonance neurography (MRN) are considered complementary to clinical and neurophysiological assessment for neuropathies.

Aims The aim of our study was to compare the accuracy of HRUS and MRN for detecting various peripheral nerve pathologies, to choose the correct investigation to facilitate prompt patient management.

Materials and Methods This prospective study was done using HRUS with $14 \mathrm{MHz}$ linear-transducer and 3 or 1.5T MR in cases referred for the assessment of peripheral nerve pathologies. Image interpretation was done using a scoring system (score 0-3 confidence level) to assess for nerve continuity/discontinuity, increased nerve signal/edema, fascicular change, caliber change, and neuroma/mass lesion. We determined the accuracy, sensitivity, and specificity of these modalities compared with the diagnostic standard determined by surgical and/or histopathological, if not performed then clinical and/or electrodiagnostic evaluation.

Results The overall accuracy of MRN was $89.3 \%$ (specificity: 66.6\%, sensitivity: 92.6\%, negative predictive value [NPV]: $57.1 \%$, positive predictive value [PPV]: 95\%) and that of HRUS was $82.9 \%$ (specificity: $100 \%$, sensitivity: $80.4 \%$, NPV: 42.8 , PPV: $100)$. The confidence level for detecting nerve discontinuity and change in nerve caliber was found to be higher on ultrasonography than magnetic resonance imaging (MRI) (100 vs. $70 \%$ and 100 vs. $50 \%$, respectively). Pathology of submillimeter caliber nerves was accurately detected by HRUS and these could not be well-visualized on MRI. Conclusion HRUS is a powerful tool that may be used as the first-line imaging modality for the evaluation of peripheral nerve pathologies, and a better means of evaluation of peripheral nerves with submillimeter caliber.
\end{abstract}

published online April 19, 2021
DOI https://doi.org/

10.1055/s-0041-1729130 ISSN 0971-3026

\footnotetext{
(C) 2021. Indian Radiological Association

This is an open access article published by Thieme under the terms of the Creative Commons Attribution-NonDerivative-NonCommercial-License, permitting copying and reproduction so long as the original work is given appropriate credit. Contents may not be used for commercial purposes, or adapted, remixed, transformed or built upon. (https://creativecommons.org/licenses/by-nc-nd/4.0/).

Thieme Medical and Scientific Publishers Private Ltd. A-12, Second Floor, Sector -2, NOIDA -201301, India
} 


\section{Key Message}

High-resolution ultrasound (HRUS) may be a better means of evaluation of peripheral nerves with submillimeter caliber than magnetic resonance imaging (MRI). MRI evaluation should be done when clinical suspicion is high and HRUS fails to diagnose any lesion, because of its superior accuracy and sensitivity in detecting nerve or muscle edema and perineural changes.

\section{Introduction}

Peripheral nerve pathologies are commonly encountered by clinicians in practice. They rely primarily on the information gained by nonanatomical tests like clinical examination, neurophysiological assessment, and on clinical history for the evaluation and management of these cases. With the use of imaging, it is possible to get spatial information, regarding the exact site and nature of pathology as well as the surrounding structures, which is crucial for further management. ${ }^{1}$ HRUS and magnetic resonance neurography (MRN) are now considered complementary to clinical and neurophysiological assessment for neuropathies and depending on the clinical question, appropriate choice needs to be made.,3 Both the modalities are unique in their respective ways, with HRUS being more comfortable for the patient, cheap, easily available, provides higher image resolution than MR but has a steep learning curve and is highly operator dependent. ${ }^{4,5}$ MRI is expensive, sometimes not comfortable for the patient, not dependent on the operator, and has a high spatial resolution. The aim of our study was to compare accuracy of HRUS and MRN for detecting various types of peripheral nerve pathologies, to choose the correct investigation to facilitate prompt patient management.

\section{Materials and Methods}

A prospective study was performed using HRUS imaging with $14 \mathrm{MHz}$ linear transducer (Siemens S2000) and Siemens MAGNETOM 3 or 1.5T MR (Siemens Healthcare, Erlangen, Germany) in cases referred for peripheral nerve pathologies. Ethical committee approval and prior patient consent were obtained. Image interpretation was done using a scoring system (score $0-3$ confidence level) to assess for nerve continuity/discontinuity, increased nerve signal/edema, fascicular change, caliber change, and neuroma/mass lesion.
Highest confidence level was denoted by score 3 and lowest by score 1 . The confidence level for each of these findings compared for both the modalities using the $z$-test and $p$-value was calculated. Patients with polyneuropathies, MR contraindication, claustrophobia, and imaging of brachial/lumbar plexus were excluded. MRN was performed using body coil (3T: 6-channel; 1.5T: 9-channel) or large flex coil (3T: 4-channel; 1.5T: 4-channel) depending upon the size of the region scanned and various sequences (T2-weighted imaging [T2WI] fat-sat, T1WI with or without contrast, three-dimensional [3D] T2WI/short-time inversion recovery Sampling Perfection with Application optimized Contrasts using different flip angle Evolutions for submillimeter resolution of nerves, and 3D diffusion-weighted PSIF) were obtained. MRs were reported by two radiologists each with more than 10 years' experience in MSK MRI. US was subsequently performed by a single fellowship-trained MSK consultant (JPS with more than 10 years' experience), who was unaware of MR findings. Radiologists were blinded to nerve conduction velocity (NCV), electromyography (EMG), and clinical details. Studies were conducted in close time intervals to exclude any error or difference in findings due to interval change in lesion characteristics. We determined the accuracy, sensitivity, and specificity of these modalities against the diagnostic standard determined by surgical and/or histopathological evaluation, if not performed then clinical and/or electrodiagnostic evaluation.

\section{Results}

Thirty-eight patients ( 25 males, 13 females), with the mean age of 43 years and a total of 47 nerves were evaluated using HRUS and MRN, against diagnostic standard determined by surgical (29\%), histopathological (10.5\%), clinical (13.1\%), and/or electrodiagnostic evaluation (47.3\%). The nerves involved included median (9), ulnar (8), radial (7), anterior interosseous (2), posterior interosseous (2), sciatic (3) common peroneal (6), sural (3), tibial (4), and others (3) like one each of spinal accessory, posterior, and medial cutaneous nerve of the forearm. Specificity of HRUS was higher (100\%) than that of MRI (66.6\%). Sensitivity of MR was higher at $92.6 \%$ versus $80.4 \%$ for HRUS $(p=0.104)$. The overall accuracy of MRN (-Table 1) was $89.3 \%$ (negative predictive value [NPV]: $57.1 \%$, positive predictive value [PPV]: $95 \%$ ) and that of HRUS was $82.9 \%$ (NPV: 42.8 , PPV: 100). Confidence level (-Table 2) for detecting nerve discontinuity and change in nerve caliber

Table 1 The overall accuracy of MRN

\begin{tabular}{|l|l|l|l|l|}
\hline & \multicolumn{2}{|l|}{ MRI } & US \\
\hline Statistic & Value & $95 \% \mathrm{Cl}$ & Value & $95 \% \mathrm{CI}$ \\
\hline Sensitivity & $92.68 \%$ & $80.08-98.46 \%$ & $80.49 \%$ & $65.13-91.18 \%$ \\
\hline Specificity & $66.67 \%$ & $22.28-95.67 \%$ & $100.00 \%$ & $54.07-100.00 \%$ \\
\hline Positive predictive value & $95.00 \%$ & $85.93-98.34 \%$ & $100.00 \%$ & \\
\hline Negative predictive value & $57.14 \%$ & $28.09-81.98 \%$ & $42.86 \%$ & $28.71-58.27 \%$ \\
\hline Accuracy & $89.36 \%$ & $76.90-96.45 \%$ & $82.98 \%$ & $69.19-92.35 \%$ \\
\hline
\end{tabular}

Abbreviations: $\mathrm{Cl}$, confidence interval; MRN, magnetic resonance neurography; US, ultrasound. 
Table 2 Confidence level for various parameters on MRI and US

\begin{tabular}{|l|l|l|l|l|}
\hline & $\boldsymbol{n}$ & MRI & US & $p$-Value \\
\hline Nerve discontinuity & 10 & $7(70.0 \%)$ & $10(100.0 \%)$ & $0.009^{\text {a }}$ \\
\hline Increased nerve signal & 13 & $13(100.0 \%)$ & $9(69.2 \%)$ & $0.033^{\text {a }}$ \\
\hline Fascicular change & 18 & $16(88.9 \%)$ & $18(100.0 \%)$ & 0.151 \\
\hline Caliber change & 14 & $8(57.1 \%)$ & $14(100.0 \%)$ & $0.007^{\text {a }}$ \\
\hline Neuroma/mass lesion & 9 & $8(88.9 \%)$ & $9(100.0 \%)$ & 0.317 \\
\hline
\end{tabular}

Abbreviations: MRI, magnetic resonance imaging; US, ultrasound. ${ }^{a} p$-Value $<0.05$, statistically significant.

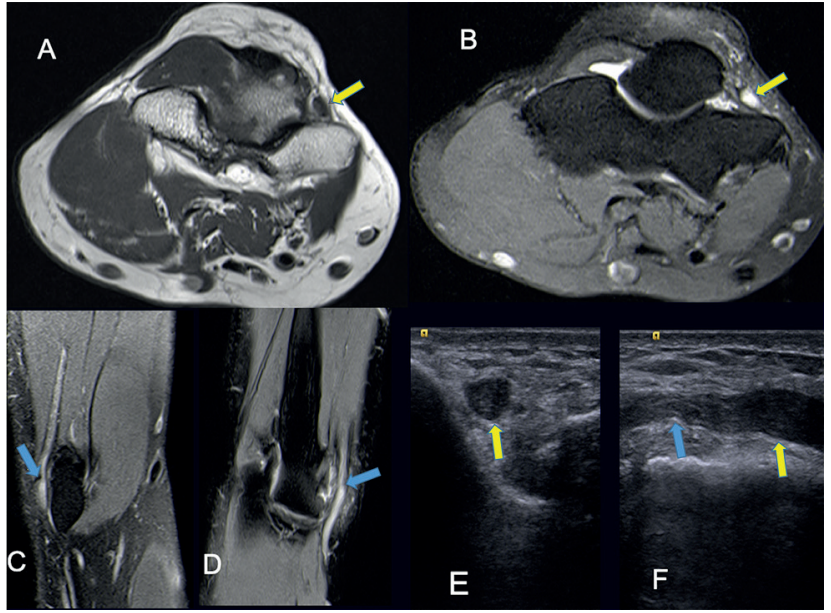

Fig. 1 Cubital tunnel syndrome (right elbow) (A, B) proximal to ulnar nerve compression, there is swelling with loss of normal fascicular pattern (A). Hyperintensity (B) is seen (arrows) on axial proton-density fat saturated images. (C, D) Reformatted three-dimensional short-time inversion recovery Sampling Perfection with Application optimized Contrasts using different flip angle Evolutions image clearly shows site of nerve compression by thickened retinaculum (blue arrow C, D, and F) with proximal increase in signal intensity of nerve. (E, F) Ultrasound images show compressed ulnar nerve with sudden increase in caliber of nerve proximal to it (yellow arrow).

(-Fig. 1) was found to be higher on US than MRI (100 vs. $70 \%$ [ $p=0.009$ ] and 100 vs. 50\% [ $p=0.007]$, respectively). MRI detected nerve/muscle edema in cases (-Fig. 2), where US had less confidence level ( $p=0.033$ ), leading to higher sensitivity of MRI. Confidence for diagnosing neuroma formation (-Fig. 3) was high for both modalities (100\% for US vs. $88.8 \%$ for MRI) with no statistically significant difference $(p=0.317)$. Pathology of submillimeter caliber nerves $(n=3)$ was accurately detected by HRUS ( - Figs. 4 and 5), and these could not be diagnosed on MRI.

\section{Discussion}

Imaging in peripheral nerve pathologies complements clinical history/examination, EMG, NCV findings by giving the spatial and morphological information of the pathology and thus influences patient management. ${ }^{1-3}$ Also, peripheral nerve imaging is helpful in patients with indeterminate findings on electrodiagnostic studies (especially postoperative patients) and in patients in whom electrodiagnostic studies are not feasible due to inaccessible nerves or with

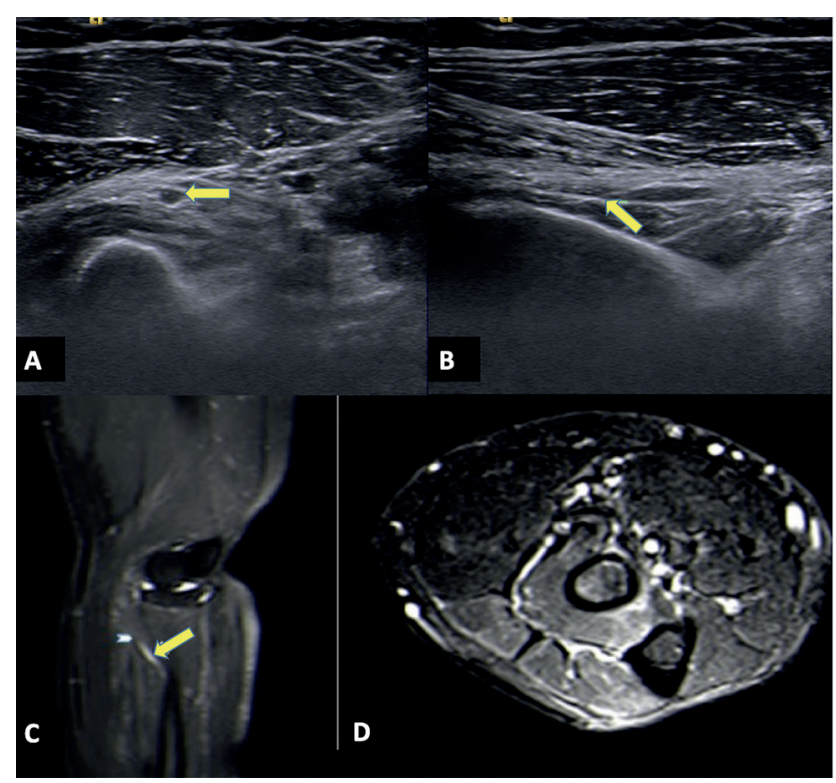

Fig. 2 Posterior interosseous nerve syndrome. Ultrasound images (A) in a 60-year-old female patient with pain in anterolateral aspect of right elbow show hypoechoic mildly thickened posterior interosseous branch of radial nerve (yellow arrow in A) with a thin echogenic constricting band (arrow in B) seen at proximal edge of supinator muscle (arcade of Frohse). Sagittal short-time inversion recovery (STIR) (C) magnetic resonance imaging (MRI) depicts the thickened hyperintense nerve (arrow head) with abrupt narrowing (yellow arrow). Axial STIR MRI (D) shows edema within supinator and extensor digitorum muscles.

dermatological conditions., ${ }^{6,7}$ With limited or indirect indications of computed tomography or radiography, peripheral nerve imaging mainly relies on HRUS or MRN and appropriate choice of imaging modality needs to be made to facilitate prompt patient management. In our study, we compared accuracy of HRUS and MRN for detecting various types of peripheral nerve pathologies. Characters like the continuity of the nerve, change in caliber, focal lesion or neuroma formation, and focal nerve edema were evaluated.

Our study has shown MRN to be more accurate in detecting the peripheral nerve pathologies ( 89.3 vs. $82.9 \%$ ) with higher negative predictive values for diagnosing the lesions. Similar findings were noted in a comparative study published by Agarwal et al, ${ }^{8}$ who also reported higher accuracy of MRI over HRUS (93.89 vs. 86.11\%) with higher negative predictive value of MRI. The confidence levels to detect pathological characters like caliber change $(p=0.007)$ and nerve 


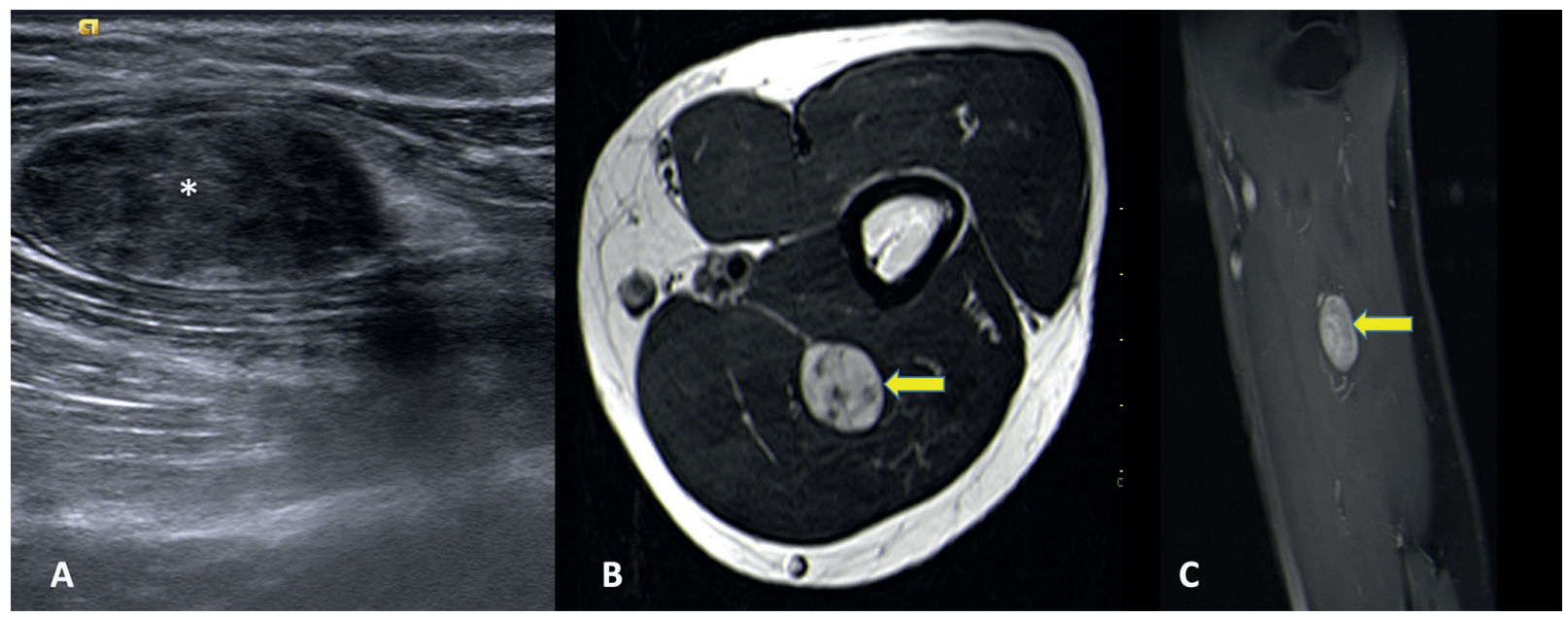

Fig. 3 Musculocutaneous nerve neurogenic tumor. Sagittal ultrasound image (A) at distal arm shows a well-defined hypoechoic lesion (asterisk) along the musculocutaneous nerve. Axial T2-weighted (B) and sagittal postcontrast T1-weighted (C) magnetic resonance imagings show the well-defined intensely enhancing oval lesion (arrow in B and C) along the course of musculocutaneous nerve between the brachialis and biceps brachii muscles.

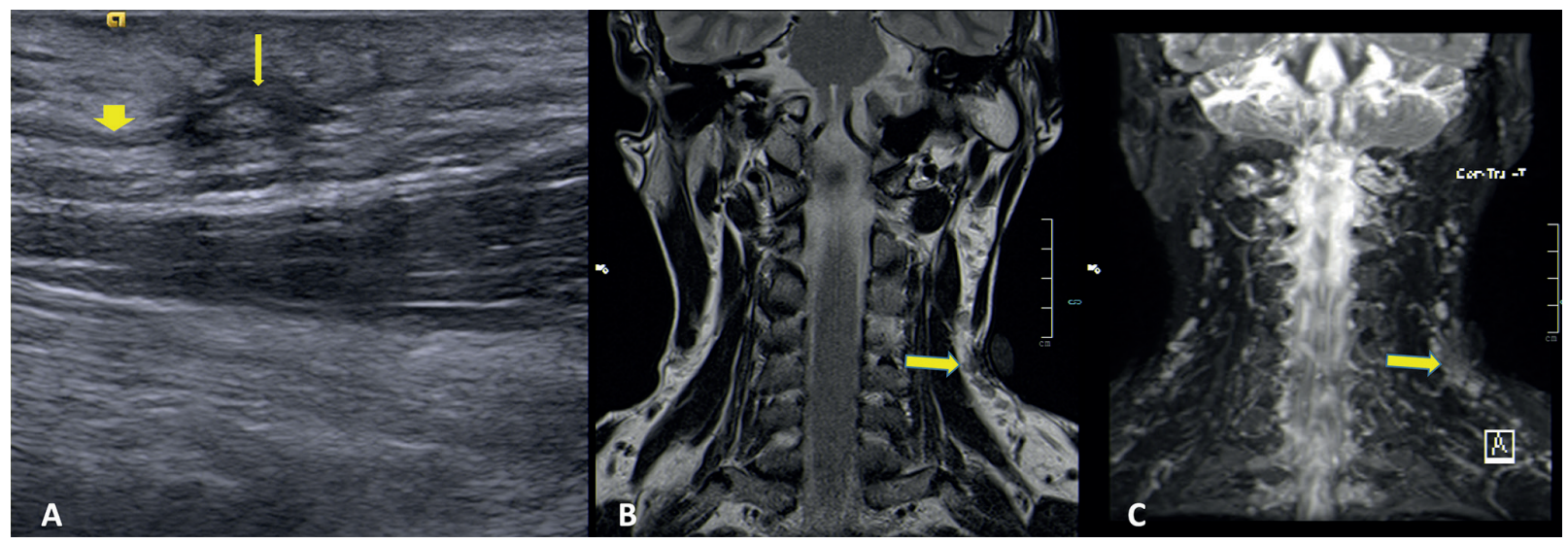

Fig. 4 Spinal accessory nerve neuroma. A 37-year-old male with weakness of left trapezius muscle following biopsy of a neck lymph node. Ultrasound image (A) shows an irregular $(1.8 \mathrm{~mm}$ ) neuroma (arrow) within the submillimeter caliber spinal accessory nerve (block arrow), which was not identifiable on magnetic resonance imaging (coronal T2 and short-time inversion recovery MIP images) with capsule at the site of lymph node biopsy (arrow).

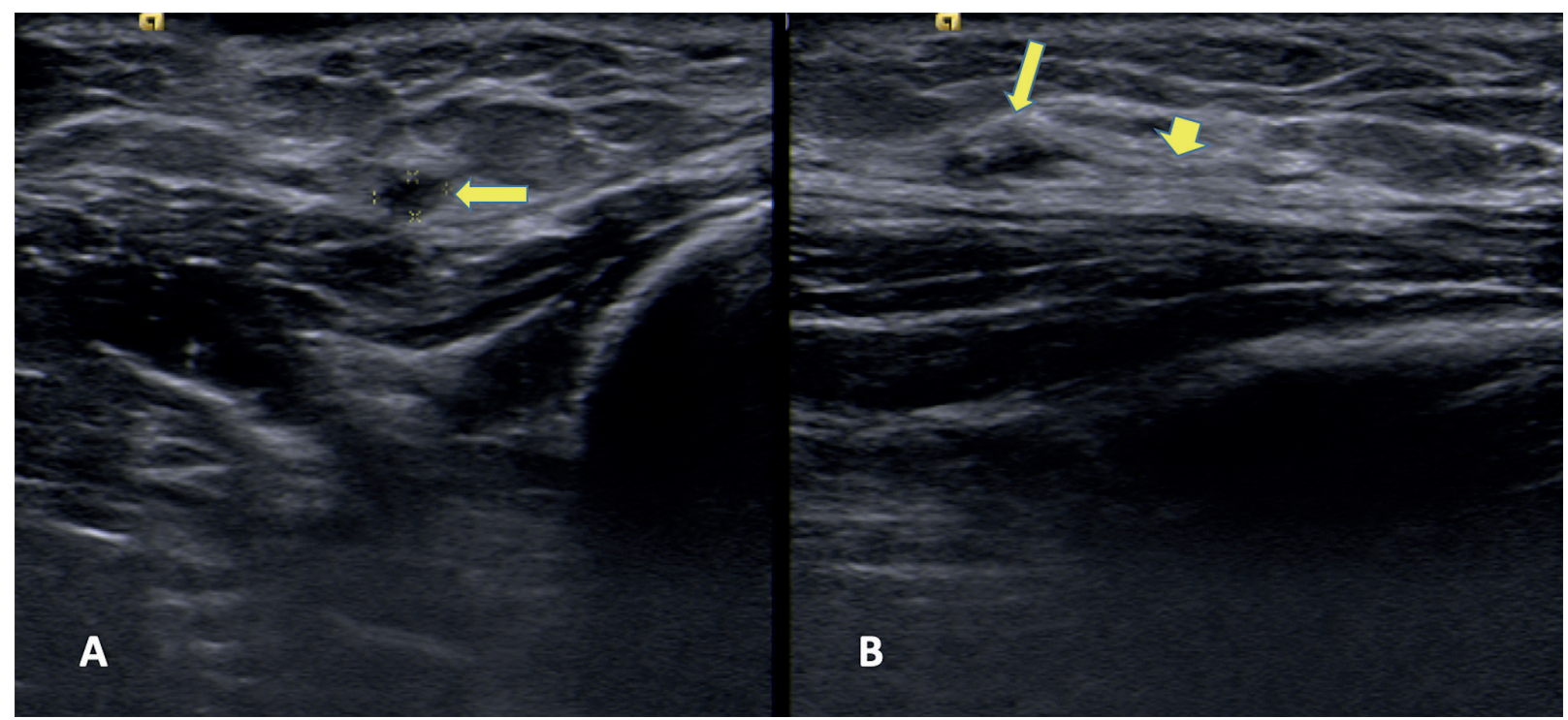

Fig. 5 End neuroma of medial cutaneous nerve of forearm ultrasound image (A: short axis, B: long axis) at the level of distal forearm in a 28-year-old male patient shows a $1.5 \mathrm{~mm}$ end neuroma (arrow) in the submillimeter caliber medial cutaneous nerve of forearm (block arrow). Magnetic resonance imaging of the same patient (images not shown) could not conclusively depict the neuroma formation. 
discontinuity $(p=0.009)$ were higher with HRUS than MRI (100 vs. $50 \%$ and 100 vs. $70 \%$, respectively) and found to be statistically significant $(p<0.05)$. Confidence level for detection of focal neuroma formation was high with both $(100 \%$ for US vs $88.8 \%$ for MRI) with no statistically significant difference. However, MRI detected nerve edema with more confidence in cases, whereas US depicted no abnormality leading to higher rates of pathological diagnosis $(p=0.033)$. Garg et $\mathrm{al}^{9}$ in their study also evaluated confidence levels for these characteristics and they had a similar impression for detecting nerve discontinuity, neuroma detection, and detection of nerve edema on MRN. However, in their study confidence level was higher for MRN in detecting change in caliber, which can probably be explained due to difference in the frequency of the US probe used in both studies. We used a 14-MHz high-resolution probe and they used 7 to $10 \mathrm{MHz}$ linear array probe.

We found MRN to be more sensitive than HRUS (92.6 vs. $80.4 \%$ ) in detecting the pathology, which is similar to what Garg et $\mathrm{al}^{9}$ found in their study. High sensitivity of MR has also been reported by Andreisek et al ${ }^{10}$ However, this was not found to be statistically significant ( $p=0.107$ ) in our study.

These observations in our study were in contradiction to the findings in study by Zaidman et $\mathrm{al}^{11}$ who reported higher sensitivity and accuracy of US over MR. This might be attributed to their study being retrospective and the authors were comparing MR reports done at various centers, in which they had no control in the acquisition of images in most of the cases, to the US performed by a single operator (the author). Also, they had access to only a few studies for review and that too were performed on 1.5T MR scanner with no known details of the sequences and protocols used.

Agarwal et al ${ }^{8}$ also reported higher specificity with MRI (86.67 vs. $80 \%$ ) with higher positive predictive values; however, in our study HRUS showed higher positive predictive value (100 vs. $95 \%$ ) with higher specificity (100 vs. $66.6 \%$ ), which can be attributed to poor image quality for MRN in few of our patients with metallic implants or movement artifacts and use of high-resolution sonographic probe. Zaidman et $\mathrm{al}^{11}$ found specificity to be similar with both modalities, while Garg et $\mathrm{al}^{9}$ have not evaluated both for the same.

Pathology of submillimeter caliber nerves (i.e., spinal accessory, posterior and medial cutaneous nerve of forearm) was accurately detected on US because of a high-frequency probe that gives submillimeter resolution. These could not be diagnosed on MRI, probably because of wider field of view imaging that made it difficult to evaluate submillimeter caliber nerves.

The study limitations were small sample size, referral bias with no control group, and the low reliability of the diagnostic standards (as only $39.5 \%$ cases are confirmed with surgery or histopathology). Statistical difference between 3 and 1.5T MRI with US neurography was not assessed, considering small sample size and it needs future research.

\section{Conclusion}

Imaging plays a crucial role in the evaluation, prognostication, and management of patients with peripheral nerve pathologies. HRUS is a powerful tool that may be used as the first-line imaging modality for the evaluation of peripheral nerve pathologies, as it is dynamic, economical, comfortable for the patients and has high confidence levels to detect pathology with a trained operator. We also conclude that for peripheral nerves with submillimeter caliber, HRUS may be a better means of evaluation than MRI and that the confidence level for detecting nerve discontinuity and change in nerve caliber is higher on HRUS. MRI evaluation should, however, be done when clinical suspicion is high and HRUS fails to diagnose any lesion, because of its superior accuracy and sensitivity in detecting nerve or muscle edema and perineural changes.

\section{Note}

Work primarily performed at Department of Radiology, Medanta-The Medicity, Gurugram, Haryana, India.

\section{Source(s) of Support}

Nil.

\section{Financial Support and Sponsorship}

Nil.

\section{Conflict of Interest}

There are no conflicts of interest.

\section{Acknowledgment}

None.

\section{References}

1 Simon NG, Talbott J, Chin CT, Kliot M. Peripheral nerve imaging. Handb Clin Neurol 2016;136:811-826

2 Gagliardo A, Toia F, Maggì F, Mariolo AV, Cillino M, Moschella F. Clinical neurophysiology and imaging of nerve injuries: preoperative diagnostic work-up and post-operative monitoring. PlastAesthet Res 2015;2:149-155

3 Kwee RM, Chhabra A, Wang KC, Marker DR, Carrino JA. Accuracy of MRI in diagnosing peripheral nerve disease: a systematic review of the literature. AJR Am J Roentgenol 2014;203(6):1303-1309

4 Brown JM, Yablon CM, Morag Y, Brandon CJ, Jacobson JA. US of the peripheral nerves of the upper extremity: a landmark approach. Radiographics 2016;36(2):452-463

5 Yablon CM, Hammer MR, Morag Y, Brandon CJ, Fessell DP, Jacobson JA. US of the peripheral nerves of the lower extremity: a landmark approach. Radiographics 2016;36(2):464-478

6 Jablecki CK, Andary MT, So YT, Wilkins DE, Williams FH. AAEM Quality Assurance Committee. Literature review of the usefulness of nerve conduction studies and electromyography for the evaluation of patients with carpal tunnel syndrome. Muscle Nerve 1993;16(12):1392-1414

7 Dellon AL. Management of peripheral nerve problems in the upper and lower extremity using quantitative sensory testing. Hand Clin 1999;15(4):697-715, x

8 Agarwal A, Chandra A, Jaipal U, et al. Can imaging be the new yardstick for diagnosing peripheral neuropathy? A comparison between high resolution ultrasound and MR neurography with an approach to diagnosis. Insights Imaging 2019;10(1):104 
9 Garg K, Aggarwal A, Srivastava DN, et al. Comparison of different sequences of MRI and ultrasonogram with nerve conduction studies in peripheral neuropathies. World Neurosurg 2017:31355-4
10 Andreisek G, Burg D, Studer A, Weishaupt D. Upper extremity peripheral neuropathies: role and impact of MR imaging on patient management. Eur Radiol 2008;18(9):1953-1961

11 Zaidman CM, Seelig MJ, Baker JC, Mackinnon SE, Pestronk A. Detection of peripheral nerve pathology: comparison of ultrasound and MRI. Neurology 2013;80(18):1634-1640 